\title{
Neonatal Foreskin Substrate Has Limitations for the Immunofluorescent Screening of Monoclonal Antibodies*
}

\author{
David T. Woodley, M.D., Therese Ernst, M.D., Melinda J. Reese, B.S., Nancy M. Ogden, B.A., \\ W. Ray Gammon, M.D., Robert A. Briggaman, M.D., and Ronald J. Falk, M.D. \\ Department of Dermatology, University of North Carolina School of Medicine, Chapel Hill, North Carolina, U.S.A.
}

Two monoclonal antibodies to type IV collagen showed a marked decrease in the labeling of the dermal-epidermal junction of neonatal foreskin while the basement membrane around dermal blood vessels was brightly stained. In contrast, these antibodies labeled the junction and dermal blood vessels with approximately equal intensity when adult skin of nonforeskin site was used as substrate. Other antibodies to matrix molecules (bullous pemphigoid antigen, epidermolysis bullosa acquisita antigen, and laminin) showed excellent staining of both the dermal-epidermal junction and dermal blood vessels in both neonatal foreskin and adult skin. Further, the ultrastructural appearance of the substrates appeared identical. The implication is that neonatal foreskin is not a good substrate to use for the routine screening of monoclonal antibodies to matrix components by indirect immunofluorescence since a "false negative" evaluation may occur. J Invest Dermatol 88:167-171, 1987

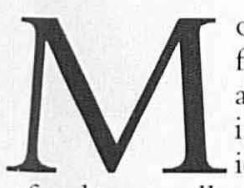

onoclonal antibodies are produced in culture by fusing sensitized spleen cells of an animal with an immortal line of $\mathrm{B}$ lymphocytes producing immunoglobulins. Raising monoclonal antibodies is labor intensive because countless numbers of culture wells must be screened for antibody production by immunologic assays such as the enzyme-linked immunoabsorbent assay (ELISA) or indirect immunofluorescent (IIF) staining of frozen tissues.

In this study, we report that two well-characterized monoclonal antibodies to human basement membrane (type IV) collagen failed to stain the dermal-epidermal junction (DEJ) of human neonatal foreskin while readily staining this structure in adult skin. This finding would suggest that when neonatal foreskin is used as substrate for IIF screening of monoclonal antibodies, it is possible to miss antibodies to components within the DEJ and inadvertently discard antibody-producing cultures based on a "false-negative" screening procedure. We recommend that several substrates, including adult human skin, be used for the IIF screening of monoclonal antibodies against skin components.

Manuscript received March 19, 1986; accepted for publication August 11, 1986.

Supported by National Institutes of Health grants AM33625, AM01540, and AM34855, and a grant from the American Cancer Society.

*This work was presented in part at the Joint International Meeting of the Society for Investigative Dermatology, Inc., and The Japanese Society for Investigative Dermatology, Washington, D.C., May 1-5.

Reprint requests to: David T. Woodley, M.D., Department of Dermatology, University of North Carolina School of Medicine, Chapel Hill, North Carolina 27514.

Abbreviations:

BMZ: basement membrane zone

DEJ: dermal-epidermal junction

EBA: epidermolysis bullosa acquisita

IIF: indirect immunofluorescence

PBS: $0.02 \mathrm{M} \mathrm{NaCl}, 0.1 \mathrm{M} \mathrm{NaPO}_{4}, \mathrm{pH} 7.4$

PEI: polyethyleneimine

\section{MATERIALS AND METHODS}

Antibodies The following antibodies were used in this study: (1) a mouse monoclonal to human type IV collagen extracted from placenta [1]; (2) a mouse monoclonal to an uncoiled region near the 7-S collagenase resistant domain of human placental type IV collagen [1]; (3) a mouse monoclonal antibody to the epidermolysis bullosa acquisita (EBA) antigen called $\mathrm{H}_{3} \mathrm{a}$ [2]; and (4) an affinity-purified sheep antilaminin polyclonal antibody [3]. In addition, 2 sera from patients with bullous pemphigoid were used that were known to stain the DEJ of human skin by IIF at dilutions of greater than 1:160 [4]. Controls included 3 normal human sera, 2 normal rabbit sera, mouse plasma, mouse serum, and $0.02 \mathrm{M}$ $\mathrm{NaCl}, 0.1 \mathrm{M} \mathrm{NaPO}_{4}, \mathrm{pH} 7.4$ (PBS).

Substrate All IIF experiments were performed with human skin. Six specimens of adult human skin of nonforeskin location (breast 2, eyelid 1, thigh 2, abdomen 1) were obtained from the Plastic Surgery Clinic. Six neonatal foreskins were obtained from the Newborn Nursery immediately following circumcision. Three adult foreskins were obtained: 2 from the Urology Service immediately following adult circumcisions and 1 sent frozen on dry ice from the National Institutes of Health. Except for this adult foreskin, all the other tissue specimens were handled identically. Tissue removed from the patient was either embedded immediately in O.C.T. cryogel (Tissue Tek, Miles Scientific, Naperville, Illinois) and frozen in liquid nitrogen ( 2 adult and 4 neonatal foreskins and 3 adult skin samples from nonforeskin sites) or placed in Eagle's basal medium containing $20 \mathrm{~mm}$ HEPES buffer $\mathrm{pH} 7.4,100 \mu \mathrm{g} / \mathrm{ml}$ of penicillin, and $100 \mathrm{U} / \mathrm{ml}$ of streptomycin that had been precooled to $4^{\circ} \mathrm{C}$ and then embedded in O.C.T. cryogel and frozen within $2 \mathrm{~h}$ ( 2 neonatal foreskins and 3 adult skin samples from nonforeskin sites).

Indirect Immunofluorescence Indirect immunofluorescent staining of the various substrates with the antibodies and sera was performed as previously described [5]. All antibodies were diluted in PBS at dilutions between $1: 10$ to $1: 1280$ in order to semiquantitate the IIF staining. The staining patterns and intensity of 
the slides were read blindly by 3 investigators (DTW, WRG, RAB). An arbitrary scale was used 0 to $4+$ intensity in which a plus or minus $( \pm)$ reading was valued at 0.5 . The readings on each substrate were averaged for each dilution of a given antibody.

Electron Microscopy Tissues for transmission electron microscopy were fixed overnight in half-strength Karnovsky's fixative at $4^{\circ} \mathrm{C}$, postfixed in osmium tetroxide, embedded in Epon 812 , and stained with uranyl acetate and lead citrate prior to examination in a JEM $100 \mathrm{~B}$ electron microscope.

To determine whether the anionic sites within the DEJ might be defective, both adult skin at nonforeskin sites and neonatal foreskin were fixed in a periodate-lysine-paraformaldehyde buffer [6] and examined ultrastructurally after incubation with an electron-dense highly cationic molecule, polyethyleneimine (PEI; Polysciences, Warrington, Pennsylvania), as described by Vernicr et al [7] and modified by Manabe and Ogawa [8]. Sections were examined on a JEM 100B electron microscope, photographed at a magnification of 30,000, and enlarged and printed under uniform standardized conditions.

\section{RESULTS}

Figure 1 and Tables I and II summarize the data. Even at high concentrations of antibody (1:10 to 1:80 dilutions), the 2 monoclonal antibodies to type IV collagen either did not stain at all or minimally stained $(\leq 0.5)$ the basement membrane within the DEJ while providing maximal $(3.5-4.0)$ fluorescent staining at the basement membrane surrounding dermal blood vessels when neonatal foreskin was used as substrate. At a 1:160 dilution, these 2 monoclonal antibodies did not label the DEJ in any of the 6 specimens of neonatal foreskin but maximally stained the basement membrane around dermal blood vessels. In contrast, antilaminin antibodies at titers between 1:10-1:800 showed no diminution of DEJ staining of neonatal foreskin compared to adult foreskin or adult skin at nonforeskin sites (Table II). The anti-
Adult Skin
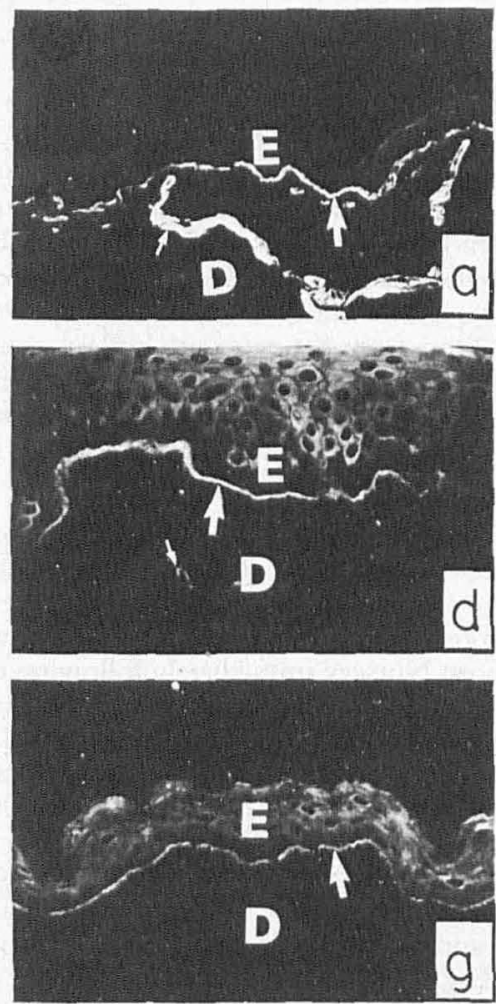

aBPA

$\alpha E B A-A$

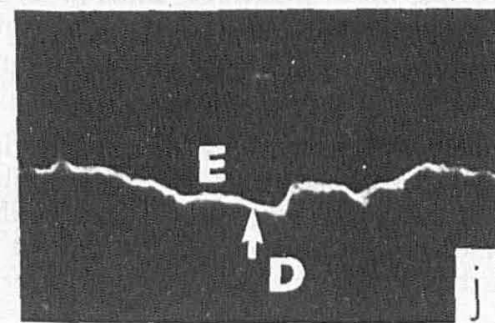

\section{Adult Foreskin}
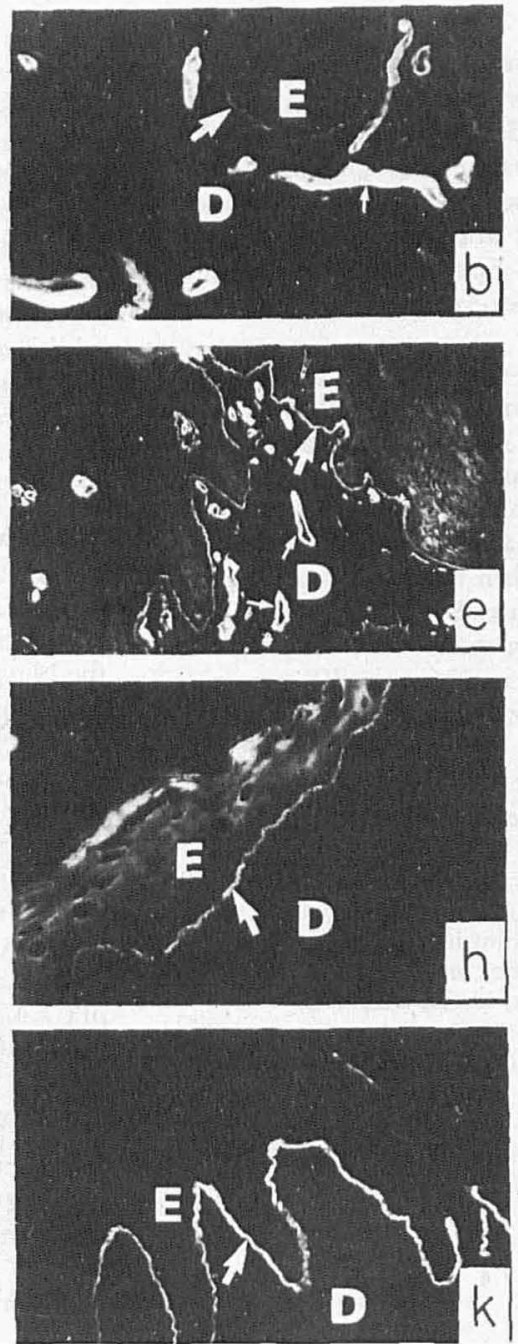

\section{Neonatal Foreskin}
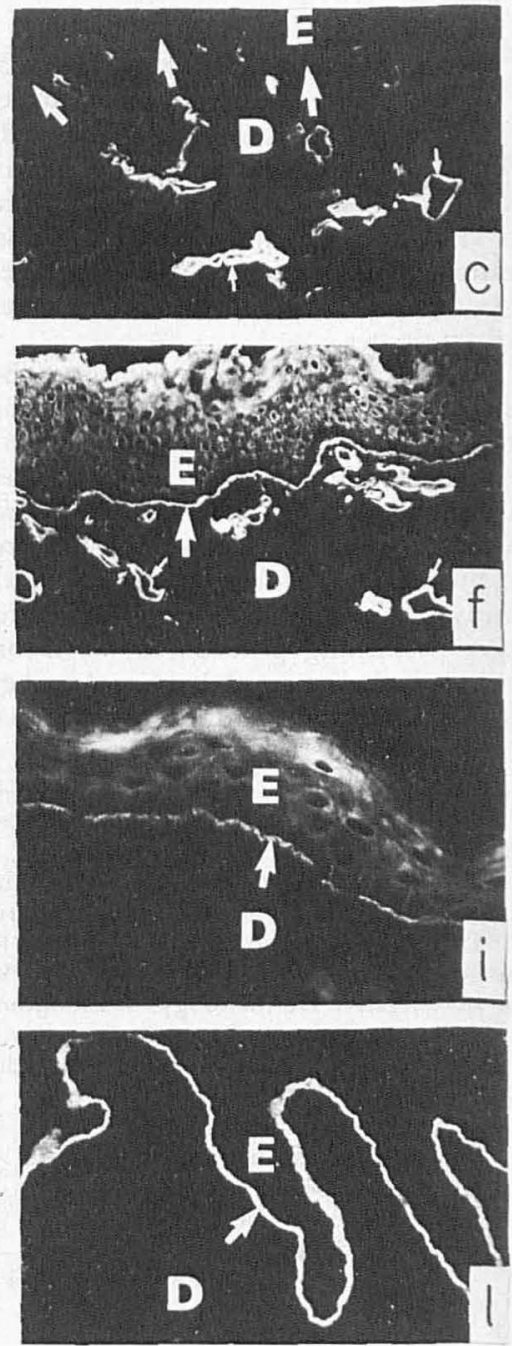

Figure 1. Indirect immunofluorescent staining of adult skin at nonforeskin sites $(a, d, g, j)$, adult foreskin $(b, e, h, k)$, and neonatal foreskin $(c, f, i, l)$ with a monoclonal antibody to type IV collagen at a 1:20 dilution $(a, b, c)$, a polyclonal antibody to laminin diluted 1:20 (d,e,f), antibodies in the serum of bullous pemphigoid patient \#2 diluted $1: 10(g, h, i)$, or a monoclonal antibody to the EBA antigen, $\mathrm{H}_{3} \mathrm{a}$, diluted $1: 10(j, k, l)$. Note that the DEJ of the neonatal foreskin is not stained with antibodies to type IV collagen vis à vis intense dermal vessel staining $(c)$. There is also less intense DEJ staining of the adult foreskin $(b)$ compared to adult skin of nonforeskin sites (a). There is no discrepant staining between the substrates with antibodies to other matrix molecules. $(E=$ epidermis, $D=$ dermis, large arrows point to the DEJ, small arrows point to dermal blood vessels.) 
Table I. Human Skin Substrate Stained With Anti-Type IV Collagen Monoclonal Antibody

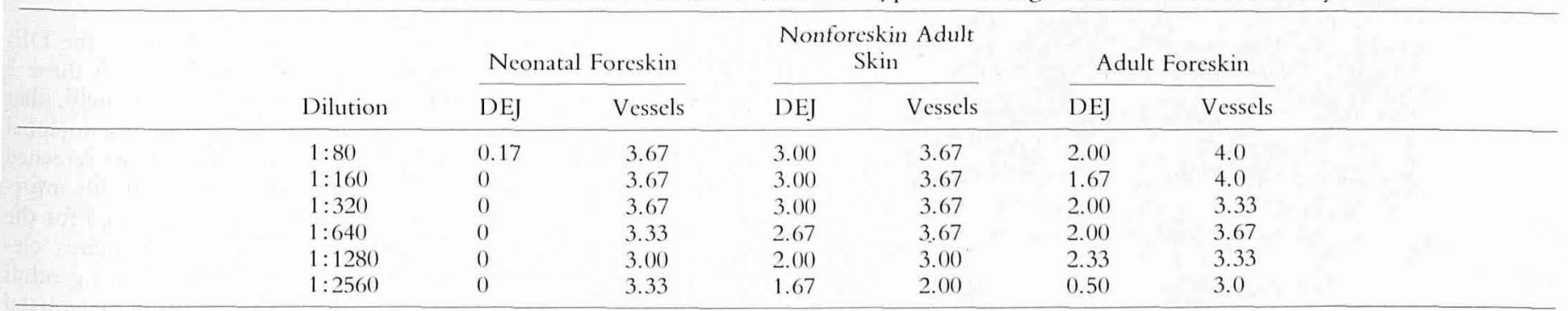

Staining was judged blindly by 3 observers using an arbitrary $0,+1,+2,+3,+4$ scale, with +4 indicating the most intense staining and 0 no staining. A reading of \pm indicating very minimal staining was scored as 0.5 . The values were summed and the means expressed in the table. There was never more than a +1 difference among the observer's readings. (DEJ = dermal-epidermal junction.)

laminin antibodies stained the DEJ of the 3 different substrates identically and showed essentially equivalent intensity of staining (never more than a +1 differential) of the basement membrane within the DEJ and around dermal blood vessels at dilutions between 1:10-1:400.

The monoclonal antibody, $\mathrm{H}_{3} \mathrm{a}$, stains only the basement membrane within the DEJ beneath stratified squamous epithelium. It does not stain dermal blood vessels since the EBA antigen is not present at this site [9]. The EBA antigen is present only in basement membrane beneath stratified squamous epithelium $[4,9]$. In contrast to the type IV monoclonal antibodies, the $\mathrm{H}_{3}$ a monoclonal showed no differential staining of the basement membrane within the DEJ of the 3 types of substrate at dilutions between 1:5-1:40. The antibody provided intense staining $(\geq 2.5)$ of the DEJ at these dilutions, and the extinguishing titers in all 3 types of substrates were between 1:80-1:160.

Like the $\mathrm{H}_{3}$ a monoclonal, both bullous pemphigoid sera stained only the DEJ and not the dermal blood vessels. Neither sera showed differential staining of the DEJ between the 3 different substrates.

Electron microscopic studies of the DEJ of the 3 different substrates showed no detectable differences. The DEJ of neonatal foreskin showed a well-organized junctional zone consisting of the plasma membrane-hemidesmosome complex, a 35-nm lamina lucida space, an intact lamina densa of approximate equal size, and a well-formed sublamina densa fibrillar zone containing anchoring fibrils and microfibrils (Fig 2).

Both adult skin at nonforeskin sites and neonatal foreskin showed excellent and identical labeling with PEI. The PEI produced 7to $9-\mathrm{nm}$ electron-dense concretions aligned along each side of the lamina densa region in the DEJ (Fig 3). These densities represent the anionic sites in the basement membrane zone (BMZ) where heparan sulfate proteoglycans (and perhaps other basement membrane proteoglycans) are localized and are thought to function as a selective permeability barrier for soluble molecules traversing the $\mathrm{BMZ}$ from the connective tissue into the epidermis $[10,11]$. In certain diseases such as nephrotic syndrome [7] and diabetes [12] the anionic sites in basement membranes are altered.

\section{DISCUSSION}

Two well-characterized monoclonal antibodies to human type IV collagen [1] showed differential staining of the basement membrane in the DEJ when neonatal foreskin, adult foreskin, and adult skin at nonforeskin sites were examined by IIF staining. Specifically, the neonatal foreskin showed markedly diminished DEJ staining when compared to adult skin of nonforeskin sites when identical dilutions of antibody were tested. Adult foreskin showed less intense DEJ staining compared to adult skin at nonforeskin sites (Fig 1, Table I). In contrast, there was no differential staining of the basement membrane within the dermal blood vessels between the 3 types of substrates when identical dilutions of the monoclonal antibodies were compared. At antibody dilutions (e.g., 1:20-1:40) where the DEJ of neonatal foreskin was not stained, the dermal blood vessels showed maximal staining (3.5-4.0). This degree of differential staining (more than a $2+$ to $3+$ difference) between the DEJ and blood vessels seen in neonatal foreskin was not observed in adult skin at nonforeskin sites where junctional and vessel staining were close to equivalent (less than a 0.75 difference) at dilutions up to 1:320 (Table I).

In contrast to the 2 monoclonals to type IV collagen, no differences in fluorescent staining of the 3 types of substrate were detected with antilaminin antibodies. Likewise, no differences were detected in the DEJ staining of the 3 substrates when a monoclonal antibody $\left(\mathrm{H}_{3} \mathrm{a}\right)$ to the EBA antigen, or 2 bullous pemphigoid sera were evaluated. Therefore, the diminished staining of the DEJ in neonatal foreskin appears limited to the 2 monoclonal antibodies to type IV collagen in our study.

This study shows that there is diminished staining of the DEJ in neonatal foreskin with only 2 monoclonal antibodies to type IV collagen, but it is possible that other newly developed monoclonal antibodies may behave similarly. Recently, investigators in the dermatology unit at the University of Rochester have found that there is variable staining of neonatal foreskin with 2 monoclonal antibodies to anchoring fibrils, major structures within the DEJ of skin. The BMZ staining of 28 foreskins with monoclonal antibodies AF1 and AF2 [13] yielded intensities from negative to

Table II. Highest Extinguishing Titer $(<0.5)$

\begin{tabular}{|c|c|c|c|c|c|c|}
\hline & \multicolumn{2}{|c|}{ Neonatal Foreskin } & \multicolumn{2}{|c|}{ Nonforeskin Adult Skin } & \multicolumn{2}{|c|}{ Adult Foreskin } \\
\hline & DEJ & Vessels & DEJ & Vessels & DEJ & Vessels \\
\hline Anti-BPA \#2 & $1: 160$ & NA & $1: 160$ & NA & $1: 160$ & NA \\
\hline Anti-EBA-A & $1: 80$ & NA & $1: 160$ & NA & $1: 80$ & NA \\
\hline Antilaminin & $1: 800$ & $1: 800$ & $1: 800$ & $1: 400$ & $1: 400$ & $1: 400$ \\
\hline Anti-Type IV & $1: 10$ & $>1: 2560$ & $>1: 2560$ & $>1: 2560$ & $>1: 2560$ & $>1: 2560$ \\
\hline Anti-7S-Type IV & $1: 20$ & $>1: 2560$ & $>1: 2560$ & $>1: 2560$ & $>1: 2560$ & $>1: 2560$ \\
\hline
\end{tabular}

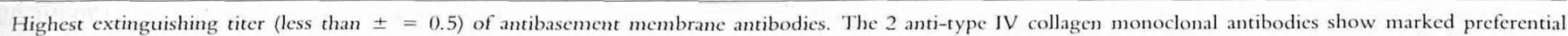

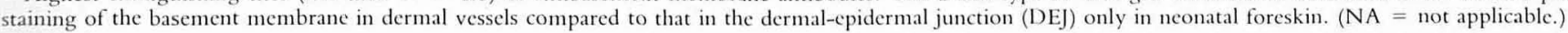




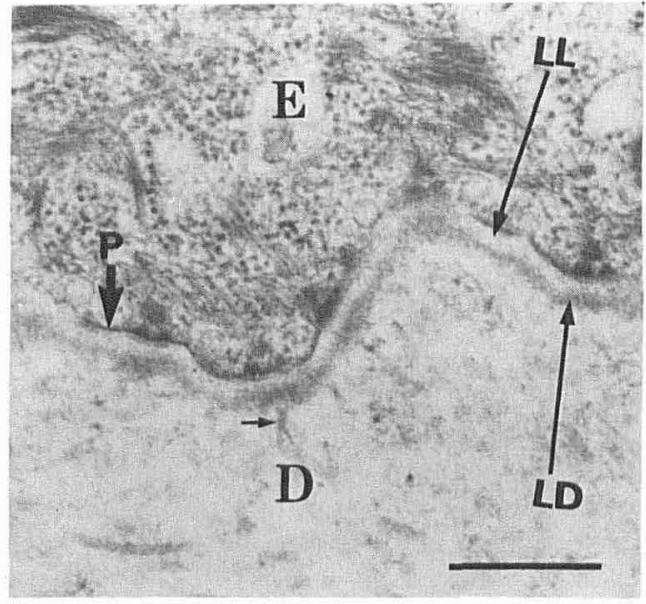

Figure 2. Electron micrograph of neonatal foreskin showing a wellformed BMZ. The epidermal cell plasma membrane, lamina lucida, lamina densa, and sublamina densa zone are all complete and identical to adult skin. $(E=$ epidermis, $D=$ dermis, $P=$ basal cell plasma membrane, $L L=$ lamina lucida, $L D=$ lamina densa-arrow points to anchoring fibril, calibration bar $=0.5 \mu \mathrm{m}$.)

$2+$ (on a scale from $0-3+$ ), with most staining at the $1+$ level (LA Goldsmith, PE McCoon, personal communication, 1986).

The lamina densa may contain laminin and heparan sulfate proteoglycan in addition to type IV collagen $[14,15]$. These 3 extremely large matrix molecules are known to have specific affinities for one another [3] and are compressed into a 35-nm electron-dense space, the lamina densa [15]. Although the DEJ of neonatal foreskin shows markedly diminished labeling with antibodies to type IV collagen, it is interesting that no morphologic perturbation of the DEJ was observed ultrastructurally (Figs $2,3)$. The ultrastructural findings vis à vis the immunofluorescent staining data might suggest that type IV collagen is present in the lamina densa of neonatal foreskin but is somehow "masked" by other matrix molecules in the region. The "masking" of matrix components associated with basement membranes has been documented previously with bullous pemphigoid antigen [16] type

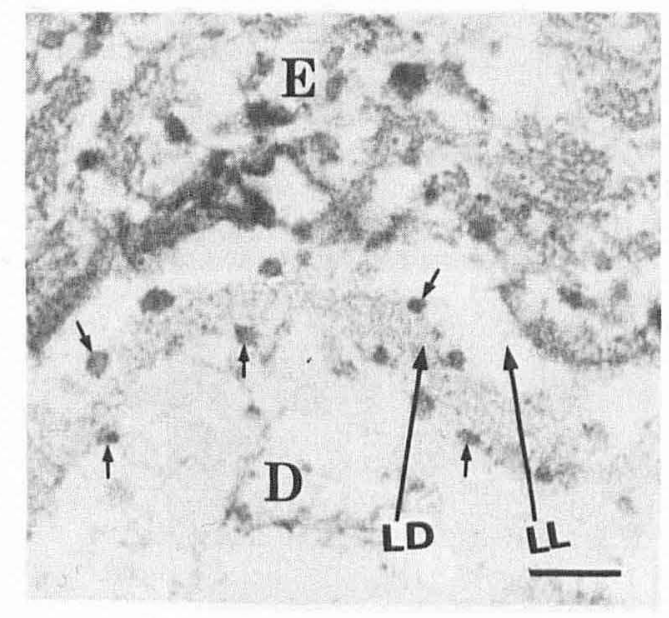

Figure 3. Electron micrograph of neonatal foreskin stained with PEI. The PEI labels anionic sites of the BMZ and is revealed as electron-dense concretions (small arrows) closely apposed to the lamina densa $(L D)$ and occupying space within the lamina lucida $(L L)$ region and the sublamina densa zone. $(E=$ epidermal basal cell, $D=$ dermis, calibration bar $=0.1$ $\mu \mathrm{m}$.
$\mathrm{V}$ collagen $[17,18]$, and glomerular basement membrane antigens [19].

Whatever the reasons for the diminished staining of the DEJ in neonatal and, to a lesser degree, adult foreskin with these 2 antibodies, the practical implications appear clear: namely, that it is possible to miss clones of hybrid cells producing monoclonal antibodies to components of the DEJ if the supernatants are screened only by IIF using neonatal foreskin as substrate. With this information in mind, we would recommend that if IIF is used for the routine screcning of monoclonal antibodies to cutancous elements, the most cautious approach would require using adult human skin at nonforeskin sites as substrate in addition to neonatal foreskin.

The authors are indebted to Dr. Jon I. Scheinman, Chief of Pediatric Neplorology at Duke University Medical Center, for the monoclonal antibodies to type IV collagen, his helpful suggestions, and his careful reading of the mamuscript. We are grateful to Dr. Stephen I. Katz, Chief of Dermatology at the National Institutes of Health for sending us tissue. We also thank Ms. Donna Foushee for the preparation of this manuscript.

\section{REFERENCES}

1. Scheinman JI, Tsai C: Monoclonal antibody to type IV collagen with selective basement membrane localization. Lab lnvest 50:101-112, 1984

2. Paller AS, Queen LL, Woodley DT, O'Keefe EJ, Gammon WR, Briggaman RA: A mouse monoclonal antibody against a newly discovered basement membrane component, the epidermolysis bullosa acquisita antigen. J Invest Dermatol 84:215-217, 1985

3. Woodley DT, Rao CN, Hassell JR, Liotta LA, Martin GR, Kleinman HK: Interactions of basement membrane components. Biochim Biophys Acta 761:278-283, 1983

4. Woodley DT, Briggaman RA, O'Keefe EJ, Inman AO, Queen LL Gammon WR: Identification of the skin basement-membrane autoantigen in epidermolysis bullosa acquisita. $N$ Engl J Med 310:1007-1013, 1984

5. Woodley DT, Sauder D, Talley MJ, Silver M, Grotendorst G, Qwarnstrom E: Localization of basement membrane components after dermal-epidermal junction separation. J Invest Dermatol $81: 149-153,1983$

6. McLean IW, Nakane PK: Periodate-lysine-paraformaldehyde fixative a new fixative for immunoelectron microscopy. J Histochem Cytochem 22:1077-1083, 1974

7. Vernier RL, Klein OJ, Sisson SP, Mahan JD, Oegema TR, Brown DM: Heparan sulfate-rich anionic sites in the human glomerular basement membrane. N Engl J Med 309:1001-1009, 1983

8. Manabe M, Ogawa H: Ultrastructural demonstration of anionic sites in basement membrane zone by cationic probes. J Invest Dermatol $84: 19-21,1985$

9. Paller AS, Queen LL, Woodley DT, Lane AT, Gammon WR, Briggaman RA: Organ specific, phylogenetic and ontogenetic distribution of the epidermolysis bullosa acquisita antigen. J Invest Dermatol 86:376-379, 1985

10. Kanwar YS, Linker A, Farguhar MG: Increased permeability of the glomerular basement membrane to ferritin after removal of GAG (heparan sulfate) by enzyme digestion. J Cell Biol 86:688-693, 1980

11. Hassell JR, Robey PG, Barrach HH, Wilczek J, Rennard SI, Martin GR: Isolation of a heparan sulfate containing proteoglycan from basement membrane. Proc Natl Acad Sci USA 77:4494-4498, 1980

12. Rohrbach DH, Hassell JR, Kleinman HK, Martin GR: Alterations in the basement membrane (heparan sulfate) proteoglycan in diabetic mice. Diabetes 31:185-188, 1982

13. Goldsmith LA, Briggaman RA: Monoclonal antibodies to anchoring fibrils for the diagnosis of epidermolysis bullosa. J Invest Dermatol 81:464-466, 1983

14. Yaoita H, Foidart JM, Katz SI: Localization of the collagenous components of skin basement membrane. J Invest Dermatol 70:191-193, 1978 
15. Lauric GW, Leblond CP, Martin GR: Localization of type IV collagen, laminin, heparan sulfate proteoglycan and fibronectin to the basal lamina of basement membrane. J Cell Biol 95:340-344, 1982

16. Remy W, Bockendahl H, Kunow H, Remy B, Stuggen G: Investigations on the binding sites of the basement membrane zone for pemphigoid antibodies in vitro. Acta Derm Venereol (Stockh) 56:423-428, 1976

17. Fitch JM, Gross J, Mayne R, Johnson-Wint B, Linsenmayer TF: Organization of collagen types I and V in the embryonic chicken cornea: monoclonal antibody studies. Proc Natl Acad Sci USA $81: 2791-2795,1984$

18. Linsenmayer TF, Fitch JM, Schmid TM, Zak NB, Gibncy E, Sanderson RD, Mayne R: Monoclonal antibodies against chicken type $V$ collagen: production, specificity, and use for immunocytochemical localization in embryonic cornea and other organs. J Cell Biol 96:124-132, 1983

19. Yoshioka K, Michael AF, Velosa J, Fish AJ: Detection of hidden nephritogenic antigen determinants in human renal and nonrenal basement membranes. Am J Pathol 121:156-165, 1985 